\title{
Development of paclitaxel-loaded liposomal nanocarrier stabilized by triglyceride incorporation
}

\author{
Soon-Seok Hong' \\ Ju Yeon $\mathrm{Choi}^{2}$ \\ Jong Oh $\mathrm{Kim}^{2}$ \\ Mi-Kyung Lee ${ }^{3}$ \\ So Hee Kim ${ }^{4}$ \\ Soo-Jeong Lim' \\ 'Department of Bioscience and \\ Bioengineering, Sejong University, \\ Seoul, ${ }^{2}$ College of Pharmacy, Yeungnam \\ University, Gyeongsan, ${ }^{3}$ College \\ of Pharmacy, Woosuk University, \\ Wanju-gun, Jeollabuk-do, ${ }^{4}$ College \\ of Pharmacy and Research Institute \\ of Pharmaceutical Science and \\ Technology, Ajou University, Suwon, \\ Republic of Korea
}

This article was published in the following Dove Press journal:

International Journal of Nanomedicine

6 September 2016

Number of times this article has been viewed

\begin{abstract}
Studies have highlighted the challenge of developing injectable liposomes as a paclitaxel (PTX) carrier, a challenge attributable to the limitations in liposomal stability caused by PTX loading. Poor stability of PTX-loaded liposomes is caused by PTX-triggered aggregation or fusion of liposomal membranes and is exacerbated in the presence of PEGylated lipid. In the present study, the effect of triglyceride incorporation on the stability of PTX-loaded/PEGylated liposomes was explored. Incorporation of a medium chain triglyceride Captex 300 into saturated phosphatidylcholine (PC)-based liposomes (1,2-dimyristoyl-sn-glycero-3-phosphocholine [DMPC]:cholesterol [CHOL]:N-(Carbonyl-methoxypolyethyleneglycol 2000)-1, 2-distearoylsn-glycero-3-phospho-ethanolamine [PE-PEG]), produced a fine, homogeneous, and membranefilterable PTX-loaded liposomes fulfilling the requirement of an injectable lipid formulation. Triglyceride incorporation also greatly inhibited the time-dependent leakage of PTX from saturated PC-based liposomes, which appears to be mediated by the inhibition of liposome fusion. In contrast, triglyceride incorporation induced the destabilization and PTX leakage of unsaturated PC-based liposomes, indicating the opposite effect of triglyceride depending on the fluidity status of PC constituting the liposomal membrane. PTX release profile and the in vitro and in vivo anticancer efficacy of triglyceride-incorporated DMPC:CHOL:PE-PEG liposomes were similar to Taxol ${ }^{\circledR}$ while the toxicity of liposomal PTX was significantly lower than that of Taxol. Taken together, triglyceride incorporation provided an injectable PTX formulation by functioning as a formulation stabilizer of PEGylated/saturated PC-based liposomes.
\end{abstract}

Keywords: paclitaxel, triglyceride, PEGylation, liposome, stability, formulation

\section{Introduction}

Paclitaxel (PTX) is one of the most effective anticancer drugs and has been clinically used for the treatment of a wide range of tumors, including breast, ovarian, lung, and head-and-neck cancers. ${ }^{1}$ PTX exhibits very low solubility in water $(<0.01 \mathrm{mg} / \mathrm{mL})$ as well as in most suitable pharmaceutical solvents, causing various formulation problems. ${ }^{2}$ Taxol $^{\circledR}$, the conventional clinical formulation of PTX consisting of Cremophor EL and ethanol, is diluted prior to administration via slow infusion to avoid PTX precipitation in the blood circulation. The presence of Cremophor EL, however, causes a number of side effects in cancer patients, including hypersensitivity reactions, nephrotoxicity, and neurotoxicity. ${ }^{2-4}$ Significant research effort has been devoted to developing an alternative, Cremophor EL-free PTX formulation. These formulations include nanoparticles, liposomes, polymeric micelles, bioconjugates, and dendrimers. ${ }^{5-9}$ Compared with Taxol, loading in liposomes achieves a significant increase in the maximum tolerated dose of PTX, a shorter infusion time, and a fewer side effects. ${ }^{10,11}$ 
Structure of liposomes allows the loading of hydrophobic drugs within the hydrophobic region of phospholipid layers. ${ }^{12}$ PTX, as a very hydrophobic drug, is also embedded within the phospholipid bilayers of liposomes, but its bulky and asymmetric nature makes liposomal loading challenging. Earlier studies have demonstrated that the maximum PTX loading content in liposomes, measured as the molar drugto-phospholipid ratio, is $\sim 3.3 \mathrm{~mol} \% \cdot{ }^{13-15}$ Attempts to load PTX into liposomes at higher percentages led to rapid PTX leakage/precipitation during storage or immediately after liposome preparation. Loading PTX in saturated phosphatidylcholine (PC)-based liposomes was shown to worsen the situation $(<1 \mathrm{~mol} \%)$, owing to the higher membrane rigidity, which hinders the penetration of PTX into the hydrophobic domain of bilayers. ${ }^{14,16}$ Moreover, PEGylation of liposomes aimed to extend the blood half-life of loaded PTX served a factor triggering the leakage of loaded PTX. ${ }^{17-19}$ Liposomal PTX formulations that are currently on the market $\left(\right.$ Lipusu $\left.^{\circledR}\right)$ or undergoing clinical trials $\left(\right.$ LEP-ETU ${ }^{\circledR}$ and EndoTag- $\left.{ }^{\circledR}\right)$ all use unsaturated PC (1,2-dioleoyl-snglycero-3-phosphocholine or EggPC) without PEGylated lipid supplementation, probably owing to these limitations in the stability of PTX-loaded liposomes. ${ }^{20-22}$ Saturated PCs, however, tend to produce liposomes that retain loaded drugs longer than unsaturated PCs, owing to the absence of oxidation-prone bonds and the higher phase-transition temperature of the resulting liposomes s $^{23-25}$ and PEGylation of liposomes extends the circulation time of loaded drugs in the bloodstream and thus increase drug accumulation in tumors ${ }^{26}$ by reducing interactions of liposomes with the reticuloendothelial system. Therefore, a formulation stabilizer enabling the PEGylated/saturated PC-based liposomes to retain PTX stably may provide an injectable PTX formulation with improved clinical performance.

We recently demonstrated that the incorporation of a small amount of oil, particularly medium- or long-chain triglycerides (MCT or LCT), into saturated PC-based membranes decreased lipid chain ordering and induced the formation of multilamellar vesicles with increased number of layers, thereby greatly improving the accommodation of hydrophobic drugs within liposomal membranes. ${ }^{27}$ Approximately 6 mol\% of PTX could be loaded in triglyceride-incorporated 1,2-dimyristoyl-sn-glycero-3phosphocholine (DMPC):cholesterol (CHOL) liposomes, a concentration of $\sim$ tenfold higher than that achieved using oil-free counterparts. However, the produced PTX-loaded liposomes were inadequate as in intravenous dosage form due to the large particle size of resultant liposomes. In addition, although the membrane fluidization by triglyceride contributed to the increased PTX loading during liposome preparation, it may hinder the storage of PTX-loaded liposomes by increasing the membrane destabilization. In the present study, we sought to explore the effect of triglyceride on the stability of PTX-loaded liposomes, particularly focused on the PEGylated/saturated PC-based ones. We herein report that triglyceride incorporation greatly improved the stability of PEGylated/saturated PC-based liposomes loaded with PTX, whereas destabilized PEGylated/unsaturated PC-based ones. As a result, triglyceride incorporation yielded a liposomal PTX formulation adequate with the physicochemical characteristics required for an intravenous lipid formulation. Finally, we show the promising potential of triglycerideincorporated liposomal nanocarriers in terms of anticancer efficacy and toxicity.

\section{Materials and methods Materials}

DMPC, DOPC, and soy-L- $\alpha-$ phosphatidylcholine (SoyPC) were purchased from Avanti Polar Lipid Inc. (Alabaster, AL, USA). N-(Carbonyl-methoxypolyethyleneglycol 2000)-1, 2-distearoyl-sn-glycero-3-phosphoethanolamine (PE-PEG) was obtained from NOF Corporation (Shibuya-ku, Tokyo, Japan). CHOL, trehalose, sucrose, and sodium salicylate were bought from Sigma-Aldrich Co. (St Louis, MO, USA). 3-(4,5-dimethylthiazol-2-yl)-2,5-diphenyltetrazolium bromide (MTT) was obtained from Amresco (Solon, OH, USA). PTX was purchased from LC Laboratories (Boston, MA, USA). Taxol was obtained from Bristol-Myers Squibb (New York, NY, USA). Captex 300 (C300), capric/caprylic triglyceride, was obtained from Abitec (Columbus, OH, USA). Labrafac $^{\text {TM }}$ Lipophile WL 1349 (Labrafac) was bought from Gattefossé (Nanterre, France). Hexyl 4-hydroxybenzoate was obtained from Tokyo Chemical Industry (Toshima, Tokyo, Japan). Acetonitrile, methanol, and tert-butyl methyl ether were high performance liquid chromatography (HPLC) grade. All other materials were of reagent grade and used without further purification.

\section{Cell lines and culture conditions}

Human H460 lung cancer cells and murine SCC7 squamous cancer cells were obtained from the American Type Culture Collection (ATCC, Manassas, VA, USA) and maintained in Roswell Park Memorial Institute 1640 medium (Welgene, Daegu, Republic of Korea). Mouse B16-F10 melanoma cells were purchased from the Korea Cell Line Bank (Seoul, Republic of Korea) and maintained in Dulbecco's Modified 
Eagle's Medium (Welgene). Each medium was supplemented with $10 \%$ heat-inactivated fetal bovine serum (Thermo Fisher Scientific, Waltham, MA, USA) and 100 units/mL penicillin/ streptomycin. Cells were grown in incubators in a humid atmosphere of $95 \%$ air and $5 \% \mathrm{CO}_{2}$.

\section{Preparation of liposomes}

Liposome dispersions were prepared by using cell disruptortype sonicator as reported in our earlier study. ${ }^{28}$ Briefly, total $40 \mu$ mole of lipid mixtures composed of PC and CHOL ( 7:1, molar ratio), with or without addition of the PE-PEG ( $5 \mathrm{~mol} \%$ of total lipid), were first dissolved in tertiary butyl alcohol. When PTX loading and/or C300 incorporation were required, $2.6 \mathrm{mg}$ of PTX and/or $2 \mathrm{mg}$ of C300, unless otherwise stated, were dissolved together with the lipid mixture in tertiary butyl alcohol. After rapid freezing at $-80^{\circ} \mathrm{C}$, mixtures were subjected to freeze-drying by a freeze dryer (FDU-1200, EYELA - Tokyo Rikakikai Co., Ltd, Miyagi, Tohoku, Japan) for 24 hours. Obtained lipid cakes were hydrated with $1 \mathrm{~mL}$ of saline, briefly vortexed and then subjected to bath sonication for 60 minutes at $37^{\circ} \mathrm{C}$ by using an ultrasonic cleaning bath (3510R-DTH, Bransonic Ultrasonics, Danbury, CT, USA). To obtain a liposomal dispersion with improved homogeneity, additional sonication for 7 minutes was performed by using a cell disruptor (Bioruptor ${ }^{\circledR}$, UCD- $^{\circledR}$ 200T, Cosmo Bio Co., Ltd, Tokyo, Japan) set at $250 \mathrm{~W}$. Unloaded/precipitated PTX was removed from PTX-loaded liposomes by immediate filtering of liposomal dispersions through $0.8 \mu \mathrm{m}$ syringe membrane filter. ${ }^{27,29}$ Entrapped PTX in liposomes was quantified by HPLC analysis as described in the following section.

\section{Physicochemical characterization of liposomes}

The mean particle size and polydispersity index (PI) of liposome dispersions were determined by dynamic lightscattering method using fiber-optics particle analyzer (FPAR-1000, Otsuka Electronics Co., Ltd, Osaka, Japan) as described in our earlier studies. ${ }^{30}$ The system was used in the auto-measuring mode. Particle size analysis data were evaluated using volume distribution to detect even a few large droplets.

For determining the PTX loading concentration, HPLC analysis was performed as described in our earlier study. ${ }^{30}$ In brief, $50 \mu \mathrm{L}$ of liposome dispersions containing PTX were freeze-dried and dissolved in $1 \mathrm{~mL}$ of methanol and centrifuged at 13,200 rpm for 10 minutes. The supernatant PTX samples were determined by using Nanospace SI-2
HPLC system (Shiseido Co., Ltd, Tokyo, Japan) equipped a mobile phase delivery pump (SP 3201) and UV-visible detector (SP 3002). The mobile phase used was a mixture of acetonitrile and distilled water $(65: 35, \mathrm{v} / \mathrm{v})$. The injection volume was $20 \mu \mathrm{L}$ and oven temperature was $40^{\circ} \mathrm{C}$. The flow rate was $1 \mathrm{~mL} / \mathrm{min}$ through a Capcellpak C18 column (UG120, Shiseido), and sample detection wavelength was $227 \mathrm{~nm}$. PTX loading efficiency (LE) was calculated using the following equation:

$$
\mathrm{LE} \%=\frac{\text { Amount of PTX loaded in liposomes }}{\text { Initial PTX amount }} \times 100
$$

The stability of PTX-loaded liposomes was evaluated by monitoring the changes of mean particle size, PI, and the PTX content remaining as loaded in liposomes during storage at $4^{\circ} \mathrm{C}$ or room temperature. The concentration of PTX retained in liposomes was determined after removing the precipitated PTX by filtration through a $0.8 \mu \mathrm{m}$ membrane filter.

The stability of PTX-loaded liposomes was also evaluated by monitoring the changes in the diameter and morphology of PTX-loaded liposomes by negative staining transmission electron microscopy (TEM) method. Samples for TEM were 70 -fold diluted with normal saline and dropped on 200 mesh copper grid coated with carbon. The liposomes were negatively stained with $2 \%$ uranyl acetate and dried samples were studied by using Tecnai G2 spirit (FEI Company, Hillsboro, OR, USA) operating at $120 \mathrm{kV}$. The samples were magnified $\sim 30,000 \times-42,000 \times$.

\section{Lyophilization of PTX-loaded liposomes}

PTX-loaded liposome dispersions were mixed with equal volume of distilled water containing $240 \mathrm{mM}$ trehalose or $500 \mathrm{mM} \mathrm{1:1} \mathrm{mixture} \mathrm{of} \mathrm{sucrose} \mathrm{and} \mathrm{trehalose} \mathrm{(molar} \mathrm{ratio).}$ The mixtures were frozen at $-80^{\circ} \mathrm{C}$ overnight and then subjected to lyophilization overnight. The lyophilized samples were rehydrated with original volume of distilled water and filtered through $0.8 \mu \mathrm{m}$ membrane filter to remove unloaded/ precipitated PTX. The liposome-retained PTX concentration, mean particle size, and PI of filtered samples were measured and quantified as described earlier.

\section{PTX release study}

The release test was performed using a Franz-type vertical diffusion cell. ${ }^{31}$ Approximately $0.5 \mathrm{~mL}$ solution containing $0.1 \mathrm{~mL}$ of liposomes diluted to $1 \mathrm{mg} / \mathrm{mL}$ as PTX and $0.4 \mathrm{~mL}$ of $1.35 \mathrm{M}$ sodium salicylate solution were applied into the donor compartment of each cell and the receptor chamber 
was filled with $\sim 5 \mathrm{~mL}$ of sodium salicylate solution ( $\mathrm{pH} 6.5$ ). Sodium salicylate was used to solubilize the leaked PTX as a hydrotropic agent as described in other studies. ${ }^{32}$ Magnetic stirring and temperature were set at $600 \mathrm{rpm}$ and $37^{\circ} \mathrm{C}$. Dialysis membrane (molecular weight cutoff 12,000-14,000, Cellu-Sep T4, Membrane Filtration Products, Inc., Texas, USA) was placed between donor compartment cell and receptor chamber. At appropriate time intervals up to 96 hours, $1 \mathrm{~mL}$ of samples were withdrawn from the receptor chamber and replaced by an equal volume of sodium salicylate solution. Samples were quantified by HPLC analysis as described in the "Physicochemical characterization of liposomes" section with slight modification: the mobile phase used was a mixture of acetonitrile and water (45:55, v/v), and oven temperature was set at $30^{\circ} \mathrm{C}$.

\section{Evaluation of anticancer activity in vitro}

In vitro anticancer activity was evaluated by MTT assay. H460 cells $\left(5 \times 10^{3}\right.$ cells/well in $0.1 \mathrm{~mL}$ medium $)$ were seeded into 96-well plates. Twenty-four hours later, cells were incubated with varying concentration of PTX as liposomal formulation or Taxol after dilution with saline. After 48 hours incubation under cell culture conditions, the growth and viability of cells were determined by using MTT assay. One hundred microliters of dimethyl sulfoxide was added to each well to dissolve any purple formazan crystals by active mitochondrial respiration. The absorbance was determined using a microplate reader (Eon, BioTek Instruments, Winooski, VT, USA) at $540 \mathrm{~nm}$.

Colony formation assay was also performed to evaluate the long-term anticancer activity. Five hundred B16-F10 cells were seeded in $60 \mathrm{~mm}$ diameter dishes and incubated for 16 hours at $37^{\circ} \mathrm{C}$ in an atmosphere containing $5 \% \mathrm{CO}_{2}$. Cells were then treated with increasing concentrations of PTX as Taxol or PTX-loaded liposomes. The culture medium was replaced with fresh medium containing PTX every 3 days. After 8 days, surviving cells forming visible colonies were counted after staining with $0.5 \%(\mathrm{w} / \mathrm{v})$ crystal violet (SigmaAldrich Co.) in $60 \%$ methanol.

\section{Evaluation of in vivo anticancer activity}

The in vivo anticancer activity of PTX-loaded liposomes and Taxol was assessed in SCC7 cell-bearing Balb/c nude mice. The protocols for the animal studies were approved by the Institutional Animal Ethical Committee (Yeungnam University, Republic of Korea), according to the guidelines for the Care and Use of Laboratory Animals. SCC7 cells $\left(2 \times 10^{6}\right)$ were subcutaneously inoculated into the right flanks of nude mice resulting in cancer xenografts. When the tumor volumes grew to $\sim 30 \mathrm{~mm}^{3}$, the antitumor study was started. Mice were randomly divided into four groups: group 1 for untreated (the control group), group 2 for empty (PTX-free) liposomes, group 3 for Taxol, and group 4 for PTX-loaded liposomes (15 mg/kg based on PTX concentration). Each sample was intravenously injected once in every 3 days to a total of four administrations. Tumor volume and body weight were measured at the scheduled time points. Tumor volume was determined using the equation $\left(L \times W^{2}\right) / 2$, where $L$ represents the longest diameter and $W$ represents the shortest diameter perpendicular to length.

\section{Hemolytic toxicity}

The protocols for the hemolytic toxicity studies were reviewed and approved by the Experimental Animal Ethical Committee of Ajou University (Suwon-si, Republic of Korea) according to the Guide for the Care and Use of Laboratory Animals. Ajou University's Institutional Animal Care Guidelines were followed. Hemolysis of red blood cells (RBCs) obtained from Sprague Dawley rats (Koatech Company, Gyeonggi-do, Republic of Korea) was used to test the potential hemolytic toxicity of Taxol and PTX-loaded liposomes. ${ }^{33,34}$ Sprague Dawley rat blood was withdrawn from the carotid artery and centrifuged at 3,500 rpm for 10 minutes to collect RBCs. RBCs were washed twice with saline and diluted in saline to obtain $2 \%$ of RBC suspension. Taxol or PTX-loaded liposomes were diluted with saline to get a final $0.3,0.6$, and $0.9 \mathrm{mg} / \mathrm{mL}$ concentration of PTX, and then $1: 1$ mixed with $2 \%$ of RBC suspension. The mixture was incubated at $37^{\circ} \mathrm{C}$ for 1 hour in water bath and stored at $4^{\circ} \mathrm{C}$ for 5 minutes to stop hemolysis. After brief centrifugation, supernatants were filtered through $0.2 \mu \mathrm{m}$ syringe membrane filter to remove the interference. The absorbance of hemolyzed solution was measured by microplate reader at $540 \mathrm{~nm}$. The absorbance of supernatant obtained after incubation of RBC with saline was regarded as $0 \%$ lysis and that with distilled water was regarded as $100 \%$ lysis. The percentage of hemolyzed cells was determined according to the following equation:

$$
\begin{aligned}
& \% \text { of hemolysis } \\
& \qquad=\frac{A_{540} \text { of sample }-A_{540} \text { of saline }}{A_{540} \text { of distilled water }-A_{540} \text { of saline }} \times 100 \text {. }
\end{aligned}
$$

\section{Statistical analysis}

Statistically significant differences between values obtained under different experimental conditions were determined using two-tailed unpaired Student's $t$-test. 


\section{Results and discussion \\ Effect of triglyceride on the physicochemical characteristics of PTX- loaded liposomes}

Liposomes were prepared using PC and CHOL (7:1, molar ratio) with or without PE-PEG and C300 supplementation (Table 1). DMPC and C300 were selected as saturated PC and oil component, respectively, based on our previously reported data. ${ }^{27}$ In the absence of triglyceride incorporation, DMPC:CHOL:PE-PEG liposomes accommodated 6.6-fold higher concentration of PTX compared to DMPC:CHOL liposomes. Our data conflict with earlier studies that reported the greatly reduced PTX loading in PEGylated liposomes compared to conventional liposomes. ${ }^{18,19}$ It is likely that inclusion of PE-PEG affects the arrangement of PCs in liposomal membranes differently depending on the fluidity status of PC (saturated/rigid PC in our study and unsaturated/fluid $\mathrm{PC}$ in other studies), thereby increasing (rigid PC) or reducing (unsaturated PC) the space available for embedding PTX.

C300 incorporation into DMPC:CHOL:PE-PEG liposomes increased the PTX loading concentration and the LE further (Table 1). More importantly, it prevented the foaming produced during liposome preparation process, which was reported to be caused by the mixed formation of micelles composed of PEGylated lipids (Figure 1A), ${ }^{35}$ and significantly reduced the mean droplet size and PI of resultant liposomes. In contrast, $\mathrm{C} 300$ incorporation into either DOPC:CHOL:PE-PEG or SoyPC:CHOL:PE-PEG liposomes increased their mean droplet sizes by 1.3 - and 1.8-fold (Table 1). The opposite effects of C300 on rigid/ saturated PC-based liposomes and fluid/unsaturated PCbased liposomes demonstrate the critical importance of optimal membrane fluidity in the production of small and homogeneous liposomes carrying PTX. It is likely that saturated PC fluidization by triglyceride increases the mobility of individual lipid molecules, thereby increasing the electrostatic repulsion between negatively charged phosphate group of PE-PEG molecules, ${ }^{11,36}$ which facilitates the production of small and homogeneous liposome dispersions.

\section{Effect of triglyceride on the formulation stability of saturated PC-based liposomes carrying PTX}

To assess the triglyceride effect on the stability of PTXloaded liposomes, time-dependent changes in the remaining PTX concentration, mean droplet size, and PI during storage were first compared between DMPC:CHOL:PE-PEG (C300(-)) and DMPC:CHOL:PE-PEG:C300 (C300(+)) liposomes. During storage at $25^{\circ} \mathrm{C}, \mathrm{C} 300(-)$, conventional liposomes exhibited a rapid increase of mean droplet size and PI, in consistent with other studies showing the formulation instability of PTX-loaded liposomes. ${ }^{13,17-19,37}$ In contrast, no significant changes were found in $\mathrm{C} 300(+)$ liposomes: after 4 days, the mean size and PI of C300(-) liposomes increased by 2.5 - and 1.8-fold while no significant changes were observed in $\mathrm{C} 300(+)$ liposomes (Figure 1B and C). These data indicate that $\mathrm{C} 300$ incorporation blocked PTXtriggered destabilization of liposomes. As a result, the rapid leakage of PTX, evident as early as the first time point (2 hours) and reaching a loss of $>60 \%$ of the initial loaded PTX after 2 days in C300-free liposomes, was greatly retarded by C300 incorporation: the leakage of PTX after a 2 and 7 days storage of $\mathrm{C} 300(+)$ liposomes was only $11 \%$ and $19 \%$ of initial loaded PTX (Figure 1D). These data demonstrate

Table I Effect of PE-PEG and C300 on the loaded PTX concentration, mean particle size, and PI of resultant liposomes

\begin{tabular}{|c|c|c|c|c|c|c|c|}
\hline \multicolumn{4}{|c|}{ Liposome composition } & \multirow{2}{*}{$\begin{array}{l}\text { Loaded PTX } \\
(\mathrm{mg} / \mathrm{mL})\end{array}$} & \multirow[t]{2}{*}{ LE (\%) } & \multirow{2}{*}{$\begin{array}{l}\text { Mean size } \\
(\mathrm{nm})\end{array}$} & \multirow[t]{2}{*}{ PI } \\
\hline DMPC ( $\mu$ mole $)$ & CHOL ( $\mu$ mole) & PE-PEG ( $\mu$ mole) & $\mathrm{C} 300$ (mg) & & & & \\
\hline 35 & 5 & 0 & 0 & $0.23 \pm 0.13$ & $8.8 \pm 5.5$ & $506 \pm 59$ & $0.251 \pm 0.029$ \\
\hline 35 & 5 & 2 & 0 & $1.52 \pm 0.24$ & $58.5 \pm 9.2$ & $438 \pm 17$ & $0.210 \pm 0.008$ \\
\hline 35 & 5 & 2 & 2 & $2.29 \pm 0.15$ & $88.1 \pm 5.8$ & $244 \pm 18$ & $0.135 \pm 0.009$ \\
\hline $35^{\mathrm{a}}$ & 5 & 2 & 0 & $1.85 \pm 0.08$ & $71.2 \pm 3.1$ & $433 \pm 17$ & $0.264 \pm 0.023$ \\
\hline $35^{\mathrm{a}}$ & 5 & 2 & 2 & $1.15 \pm 0.03$ & $44.2 \pm 1.2$ & $556 \pm 33$ & $0.207 \pm 0.005$ \\
\hline $35^{b}$ & 5 & 2 & 0 & $1.17 \pm 0.10$ & $45.0 \pm 3.8$ & $321 \pm 42$ & $0.167 \pm 0.012$ \\
\hline $35^{b}$ & 5 & 2 & 2 & $1.43 \pm 0.08$ & $55.0 \pm 3.1$ & $564 \pm 127$ & $0.236 \pm 0.013$ \\
\hline
\end{tabular}

Notes: The indicated amount of each component was used to produce I $\mathrm{mL}$ of liposomal dispersions. Data are presented as mean \pm SD ( $=3$ ). a ${ }^{\mathrm{D} O P C}$ was used instead of DMPC; 'SoyPC was used instead of DMPC.

Abbreviations: C300, Captex 300; CHOL, cholesterol; DMPC, I,2-dimyristoyl-sn-glycero-3-phosphocholine; DOPC, I,2-dioleoyl-sn-glycero-3-phosphocholine; LE, loading efficiency; PE-PEG, N-(Carbonyl-methoxypolyethyleneglycol 2000)-I, 2-distearoyl-sn-glycero-3-phosphoethanolamine; PI, polydispersity index; PTX, paclitaxel; SD, standard deviation; SoyPC, soy-L- $\alpha$-phosphatidylcholine. 
A
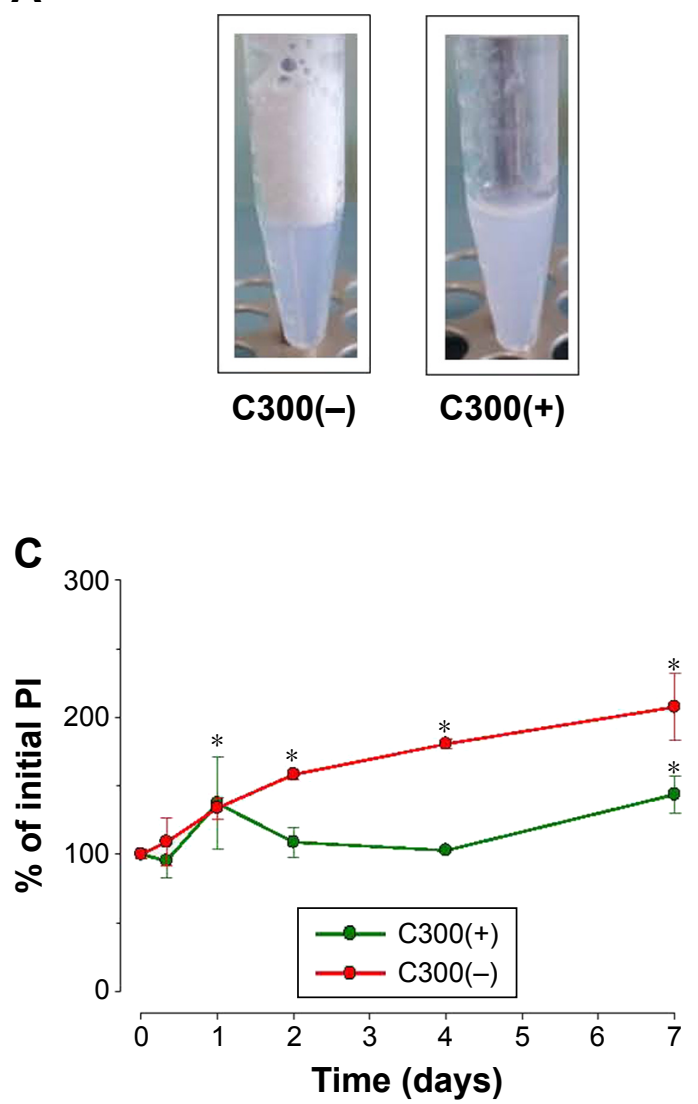

B

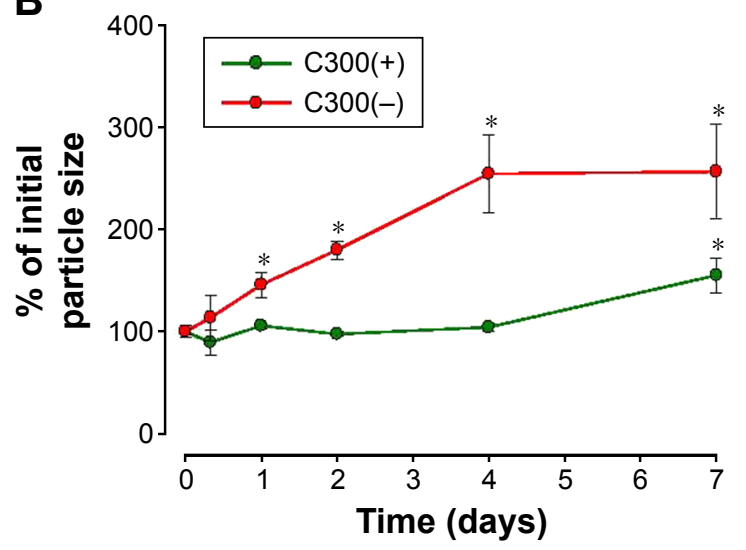

D

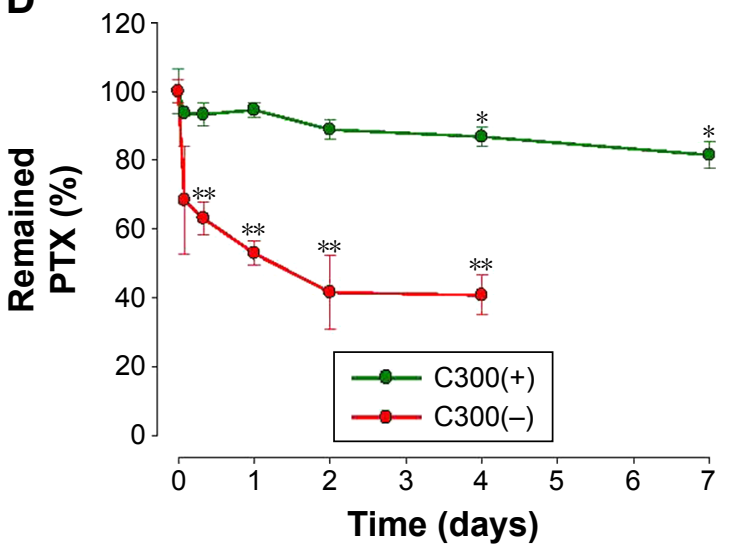

Figure I Effect of C300 incorporation on the appearance and stability of PTX-loaded liposoems.

Notes: Effect of C300 incorporation on (A) the extent of foaming during liposomal preparation process and (B-D) time-dependent changes of (B) mean particle size, (C) PI, and (D) the loaded PTX concentration of PEGylated/saturated PC-based liposomes. Liposomes were prepared with 35:5:2 mixture of DMPC:CHOL:PE-PEG with or without incorporation of $2 \mathrm{mg}$ of $C 300$. Data are expressed as mean $\pm S D(n=3)$. Significant differences are indicated by $* P<0.05 ; * * P<0.005$ compared to initial condition.

Abbreviations: C300, Captex 300; CHOL, cholesterol; DMPC, I,2-dimyristoyl-sn-glycero-3-phosphocholine; PE-PEG, N-(Carbonyl-methoxypolyethyleneglycol 2000)-I, 2-distearoyl-sn-glycero-3-phosphoethanolamine; PI, polydispersity index; PTX, paclitaxel; SD, standard deviation.

that C300 incorporation stabilizes PEGylated/saturated PCbased liposomes carrying PTX very efficiently.

The effect of triglyceride incorporation on the changes of size and morphology of PTX-loaded liposomes was visualized by obtaining TEM images. Prior to separating PTX-loaded/C300(-) liposomes from unentrapped PTX after liposomal preparation, rod-like PTX crystals were found mixed with spherical liposomal vehicles (Figure 2A). A small portion of PTX-loaded/C300(-) liposomes started to fuse and aggregate at 1 day after preparation/separation (Figure 2B) and formed very large fused/aggregated vehicles mixed with rod-like PTX crystals after 7-day storage (Figure 2C). In contrast, the sizes and morphology of PTX-loaded/C300(+) liposomes did not alter under the same condition. Neither fused vehicles nor PTX crystals were found (Figure 2D and E). Taken together, these results suggest that incorporation of C300 within phospholipid bilayer contributed to preventing the fusion/aggregation of saturated
PC-based/PEGylated liposomes, thereby greatly retarding the PTX leakage/precipitation.

Based on our recent findings that the incorporation of MCT or LCT commonly decreases the lipid chain ordering of liposomal membranes, ${ }^{27}$ we next investigated whether the stabilizing effect is also observed in case of other triglycerides. Either Labrafac (MCT) or soybean oil (LCT) also improved PTX retention in DMPC:CHOL:PE-PEG liposomes (Figure 3A), demonstrating that the stabilizing effect was commonly shared by MCT or LCT. Triglyceride incorporation may provide optimal membrane fluidity to PEGylated/ saturated PC-based liposomes, thereby maximizing the steric stabilization of liposomes by PEGylated lipids.

Since PTX is somewhat soluble in triglycerides, there is a possibility that the PTX-retaining effect of triglycerideincorporated DMPC:CHOL:PE-PEG liposomes is associated with the ability of triglyceride to serve as a PTX reservoir. In this case, increasing triglyceride content would increase 
A

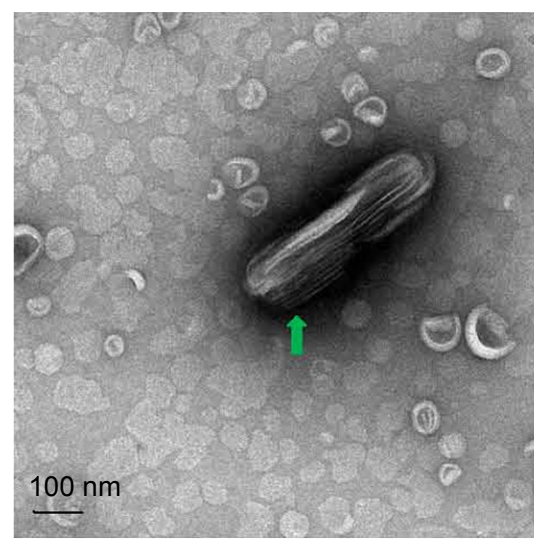

\section{C2}

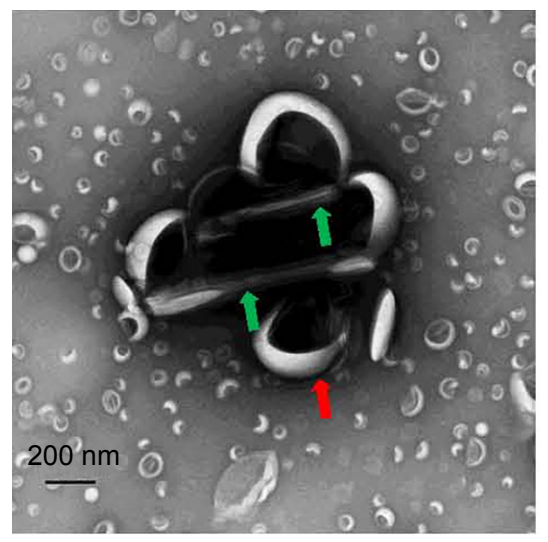

B

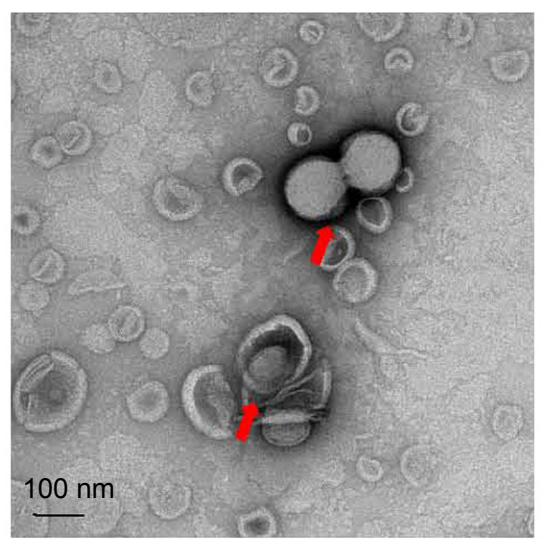

D

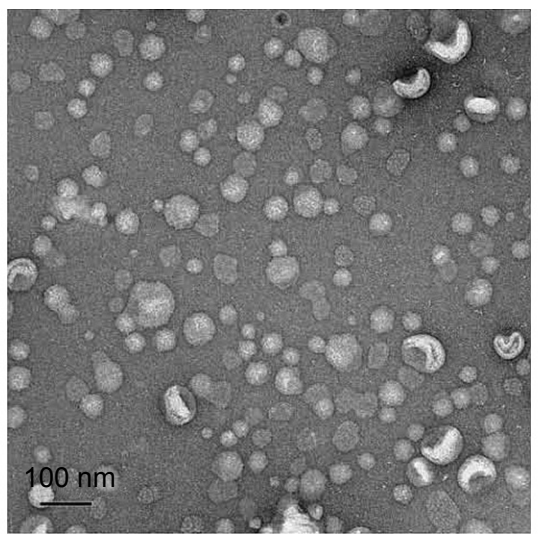

C1

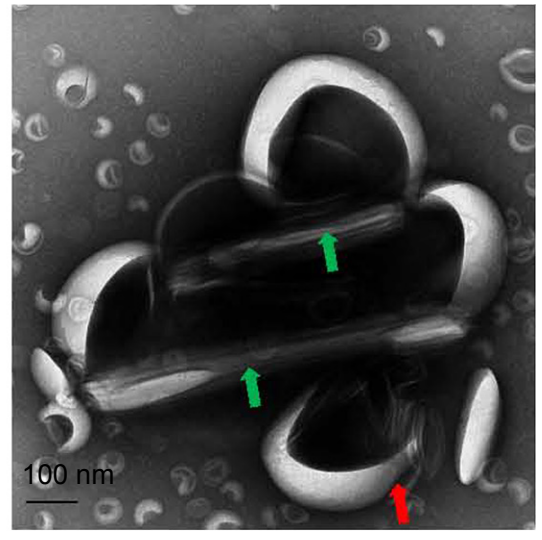

E

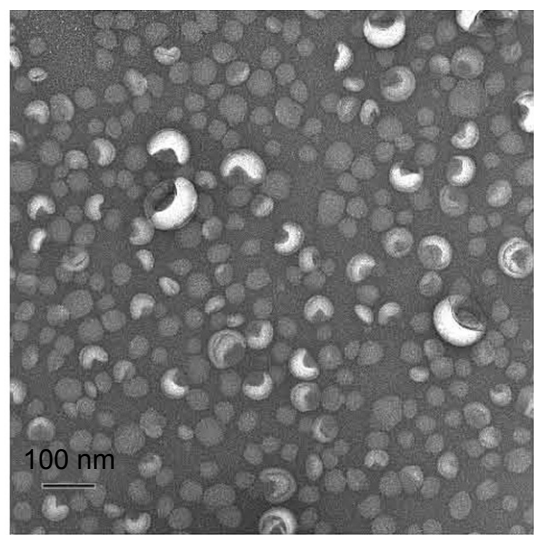

Figure 2 TEM analysis of PTX-loaded liposomes.

Notes: TEM images of (A-C) PTX-loaded/C300(-) DMPC:CHOL:PE-PEG liposomes prior to separating unloaded PTX (A), stored for I day at $4^{\circ} \mathrm{C}(\mathbf{B})$ and 7 days at $25^{\circ} \mathrm{C}(\mathbf{C}),(\mathbf{D}, \mathbf{E})$ PTX-loaded/C300(+) DMPC:CHOL:PE-PEG liposomes stored for I day at $4^{\circ} \mathrm{C}(\mathbf{D})$ and 7 days at $25^{\circ} \mathrm{C}$ (E). Rod-like PTX crystals and the fusion or aggregation of liposomes were indicated green and red arrow, respectively. TEM images in (C) were shown using both 100 (CI) and $200 \mathrm{~nm}(\mathbf{C 2})$ scale for comparison. Abbreviations: C300, Captex 300; CHOL, cholesterol; DMPC, I,2-dimyristoyl-sn-glycero-3-phosphocholine; PE-PEG, N-(Carbonyl-methoxypolyethyleneglycol 2000)-I, 2-distearoyl-sn-glycero-3-phosphoethanolamine; PTX, paclitaxel; TEM, transmission electron microscopy.
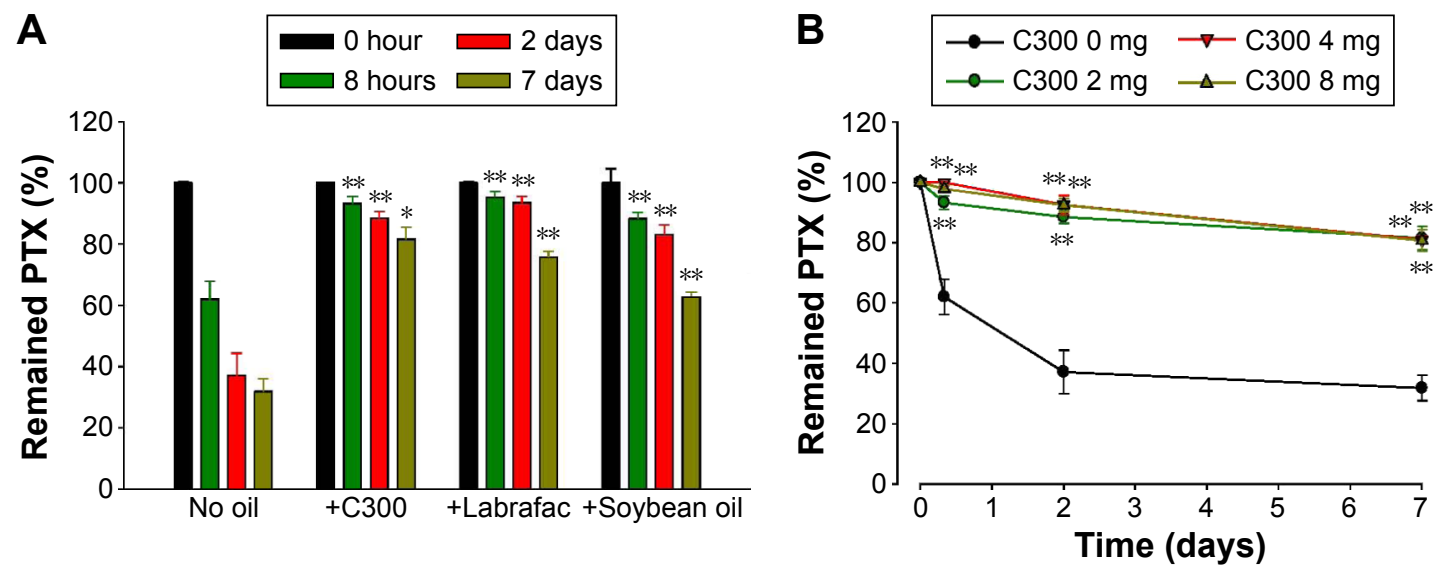

Figure 3 Effect of (A) oil type and (B) oil content on the time-dependent changes of loaded PTX concentration of liposomes.

Notes: Liposomes were prepared with 35:5:2 mixture of DMPC:CHOL:PE-PEG with or without incorporation of 2 mg of each oil (A) and indicated amount of C300 (B). Data are expressed as mean $\pm S D(n=3)$. Significant differences are indicated by $* P<0.05 ; * * P<0.005$ compared to oil-free liposomes $(\mathbf{A})$ and initial condition $(\mathbf{B})$.

Abbreviations: C300, Captex 300; CHOL, cholesterol; DMPC, I,2-dimyristoyl-sn-glycero-3-phosphocholine; PE-PEG, N-(Carbonyl-methoxypolyethyleneglycol 2000)-I, 2-distearoyl-sn-glycero-3-phosphoethanolamine; PI, polydispersity index; PTX, paclitaxel; SD, standard deviation. 
the PTX-retaining ability of triglyceride-incorporated liposomes further. However, when liposomes were prepared with increasing C300 concentration (Figure 3B), the remained PTX inside of liposomes during storage was very similar regardless of $\mathrm{C} 300$ content. The incorporation of $\mathrm{C} 300$ at $\sim 2 \mathrm{mg} / \mathrm{mL}$ in liposomes was sufficient to retard the PTX precipitation, suggesting that the triglyceride effect was saturated at a content required to fluidize the DMPC:CHOL liposomes. ${ }^{27}$

\section{Effect of triglyceride on the formulation stability of unsaturated PC-based liposomes carrying PTX}

To investigate the effect of triglyceride incorporation on the unsaturated PC-based liposomes, DOPC- or SoyPC-based liposomes were prepared in the absence or presence of $\mathrm{C} 300$.
C300 incorporation greatly induced the time-dependent increase of mean droplet size of both of DOPC- and SoyPCbased liposomes during storage: after 7 days storage, 3.5and 4.8-fold increase in the particle size were observed in DOPC:CHOL:PE-PEG:C300 and SoyPC:CHOL:PEPEG:C300 liposomes in contrast to 1.4- and 1.2-fold increase in corresponding $\mathrm{C} 300(-)$ liposomes, respectively (Figure 4A). Up to 7 days storage, $>80 \%$ of initial loaded PTX was retained in either DOPC- or SoyPC-based liposomes whereas DOPC:CHOL:PE-PEG:C300 and SoyPC:CHOL:PE-PEG:C300 liposomes already lost 44\% and $78 \%$ of initial loaded PTX only after 2- and 1-day storage, respectively (Figure 4B). These data demonstrate that C300 incorporation triggered the fusion/aggregation of PTX-loaded liposomes, causing rapid PTX leakage in unsaturated PC-based liposomes. Therefore, triglyceride
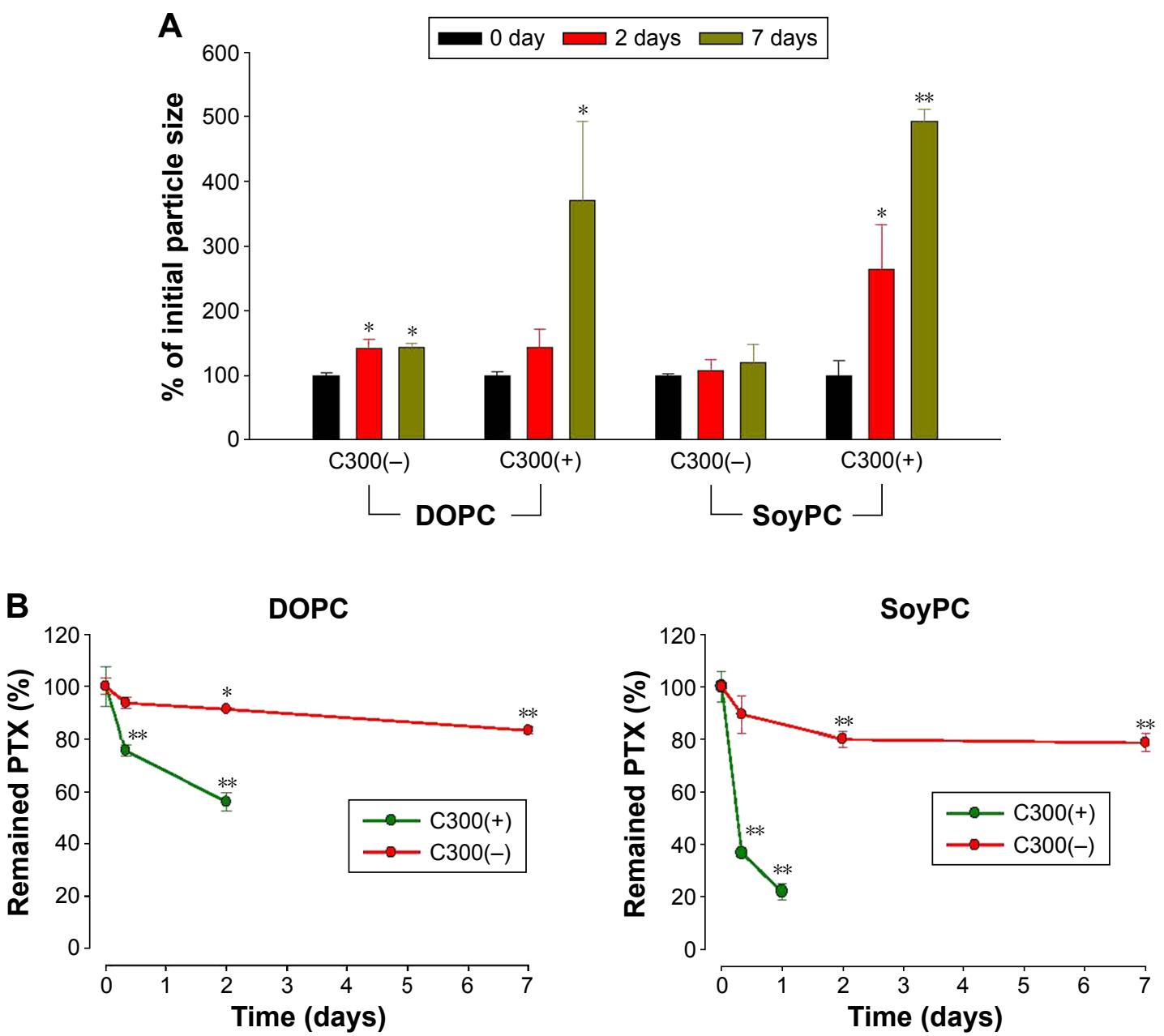

Figure 4 Effect of $\mathrm{C} 300$ on the time-dependent changes of $(\mathbf{A})$ mean particle size and the remained PTX concentration (B) of unsaturated PC-based liposomes. Notes: DOPC:CHOL:PE-PEG or SoyPC:CHOL:PE-PEG liposomes prepared with or without $\mathrm{C} 300$ ( $2 \mathrm{mg}$ per $42 \mu \mathrm{mole}$ lipid) were stored at $25^{\circ} \mathrm{C}$ for indicated period. Each point represents the mean $\pm S D(n=3)$. Significant differences are indicated by $* P<0.05 ; * * P<0.005$ compared to initial condition.

Abbreviations: C300(-), Captex 300-free liposomes; C300(+), Captex 300-incorporated liposomes; CHOL, cholesterol; DOPC, I,2-dioleoyl-sn-glycero-3-phosphocholine; PC, phosphatidylcholine; PE-PEG, N-(Carbonyl-methoxypolyethyleneglycol 2000)-I, 2-distearoyl-sn-glycero-3-phosphoethanolamine; PTX, paclitaxel; SoyPC, soy-L- $\alpha$ phosphatidylcholine; SD, standard deviation. 
incorporation exhibited opposite effect on saturated PCand unsaturated PC-liposomes carrying PTX: triglyceride functioned as a formulation stabilizer in saturated PCbased liposomes but as a destabilizer in unsaturated PCbased liposomes.

\section{Physicochemical evaluation of an injectable PTX formulation obtained by triglyceride incorporation}

Sterile filtration process is often required to obtain a liposomal formulation for injection. When $\mathrm{C} 300(-)$ liposomes were subjected to a membrane-filtration $(0.2 \mu \mathrm{m}$ pore $)$ through a syringe filter, they could be very easily filtered probably owing to the increased fluidity provided by $\mathrm{C} 300$. Moreover, it was unnecessary to perform tedious preextrusion steps to improve the homogeneity of liposomal dispersions to facilitate the filtration process. The mean particle size, PI, and loaded PTX concentration of liposomes remained unchanged by filtration and the filtered liposome dispersions exhibited physicochemical characteristics similar to parenteral emulsion (200-400 nm of droplet size and $0.100-0.250$ of PI) ${ }^{38}$ It demonstrates that triglyceride incorporation into PEGylated/saturated PC-based liposomes enabled the production of liposomal PTX formulation fulfilling the requirements of injectable lipid formulation.

Long-term stability of filtered C300(+) liposomes was next evaluated in terms of changes of mean droplet size and PI of liposomes during storage at $4^{\circ} \mathrm{C}$ in comparison of conventional $\mathrm{C} 300$-free liposomes. Both parameters remained constant for $\mathrm{C} 300(+)$ during storage at $4^{\circ} \mathrm{C}$ for 8 weeks, indicating no aggregation. In contrast, both parameters gradually increased for $\mathrm{C} 300(-)$ in a time-dependent manner (Figure $5 \mathrm{~A}$ and B). As a result, $\cong 90 \%$ PTX was
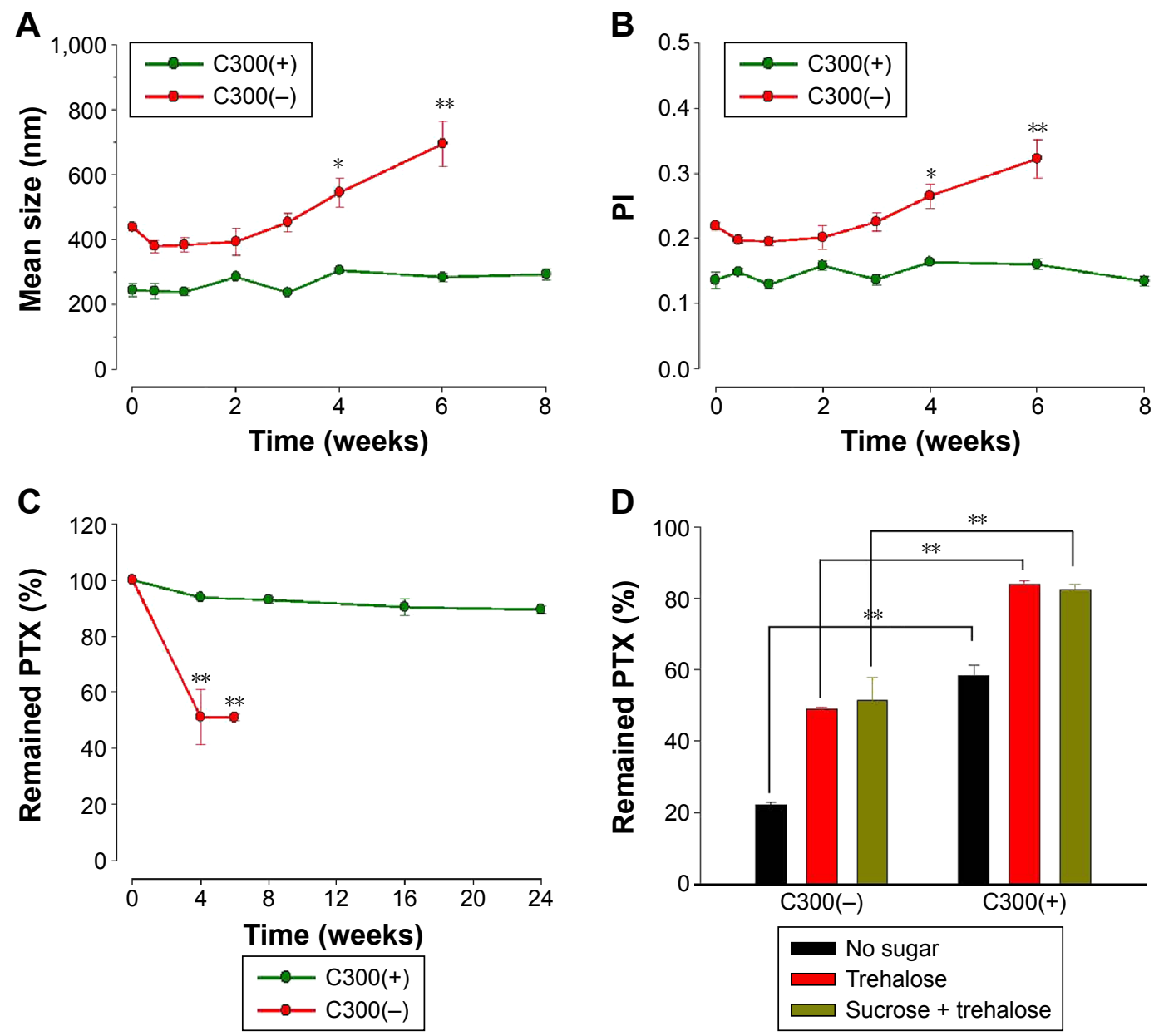

Figure 5 Long-term stability of triglyceride-incorporated/saturated PC-based liposomes.

Notes: Long-term stability of triglyceride-incorporated/saturated PC-based liposomes assessed as changes in mean droplet size (A), PI (B), and remained PTX concentration (C) of liposomes. $* P<0.05$; $* * P<0.005$ compared to initial condition. (D) Effects of triglyceride incorporation on the remained PTX in freeze-dried liposomes. PTX-loaded liposomes were prepared with a 7:1:0.4 of DMPC:CHOL:PE-PEG mixture with or without C300 (2 mg per $42 \mu$ mole lipid). Each point represents the mean \pm SD ( $=3$ ). Abbreviations: C300(-), Captex 300-free liposomes; C300(+), Captex 300-incorporated liposomes; CHOL, cholesterol; DMPC, I,2-dimyristoyl-sn-glycero-3-phosphocholine; PE-PEG, N-(Carbonyl-methoxypolyethyleneglycol 2000)-I, 2-distearoyl-sn-glycero-3-phosphoethanolamine; PTX, paclitaxel; SD, standard deviation; PI, polydispersity index. 
retained in $\mathrm{C} 300(+)$ liposomes up to 24 weeks whereas $\sim 48 \%$ PTX was already lost from C300(-) liposomes after 4 weeks (Figure 5C). Considering that the particle size and PI of parenteral emulsions mentioned earlier, C300(-) liposomes were injectable up to 2.3-week storage while C300(+) liposomes were injectable up to 24 weeks. The shelf-life of liposomes determined based on the $\geq 90 \%$ initial loaded drug concentration was $<1$ week for C300(-) liposomes and $\geq 24$ weeks for $\mathrm{C} 300(+)$ liposomes. Therefore, triglyceride incorporation in PEGylated/saturated PC-based liposomes greatly improved the storage stability of injectable liposomal formulation of PTX.

Considering that liposomal PTX formulations currently in the market or under clinical trials are freeze-dried to prevent PTX precipitation during storage, we also examined whether the triglyceride incorporation affects the liposomeretained PTX content after freeze-drying. Trehalose or sucrose:trehalose mixture was chosen as a lyoprotectant according to the optimal lyoprotecting condition reported by Kannan et al. ${ }^{39}$ Regardless of lyoprotectant addition, $\mathrm{C} 300(+)$ liposomes retained higher PTX after freeze-drying compared to $\mathrm{C} 300(-)$ liposomes: after lyophilization, the concentration of PTX retained in C300-incorporated liposomes was decreased by $42 \%$ and $16 \%$ of initial loaded PTX in the absence or presence of lyoprotectant whereas that in $\mathrm{C} 300$-free liposomes was decreased by $77 \%$ and $50 \%$ under the same conditions (Figure 5D). Taken together, it appears that $\mathrm{C} 300$, in cooperation with the lyoprotectant, contributed to maintain the membrane integrity of liposomes during lyophilization.

\section{Pharmacological evaluation of an injectable PTX formulation obtained by triglyceride incorporation}

An in vitro PTX-release study was performed to compare C300-incoporated liposomal formulation developed in the present study with Taxol, a commercial PTX product. Regardless of formulations, PTX showed a biphasic pattern of release, with an early rapid release during the first 0-48 hours, followed by a slower release (Figure 6A). There were no significant differences in the PTX release kinetics between two PTX formulations: both formulations released $\sim 60 \%$ of drug by $\sim 96$ hours under conditions where release was accelerated by sodium salicylate. ${ }^{40}$ These results indicate that PTX release from liposomes were slow comparable to that of Taxol. Particulate carriers that slowly release drug are beneficial for formulations of a drug with limited aqueous solubility since rapid release might cause drug precipitation after intravenous administration.

To assess the formulation effect on the anticancer efficacy of PTX, we compared the antiproliferative effects of liposomal PTX and Taxol on two different cancer cell lines. PTX inhibited the proliferation of human H460 lung cancer cells in a concentration-dependent manner (Figure 6B). After a 48-hour treatment, the concentration of PTX in Taxol and $\mathrm{C} 300(+)$ liposomes that inhibited growth of cells by $50 \%$ $\left(\mathrm{IC}_{50}\right)$ was 21 and $14 \mathrm{nM}$, indicating that the antiproliferative effects of PTX loaded in liposomes were similar to those of Taxol. PTX-free, empty C300(+) liposomes did not affect the cell growth, indicating that they are not cytotoxic. In addition, the anticancer efficacy of $\mathrm{C} 300(+) /$ non-PEGlayted liposomes did not differ from that of $\mathrm{C} 300(+) /$ PEGylated liposomes, implying that the PEGylation does not affect the anticancer activity of PTX under our experimental conditions (data not shown). To assess the effect of treatment for a longer period, we performed colony-formation assays using B16-F10 cells in which treatments were repeated every 3 days. As shown in Figure 6C, PTX in the two formulations decreased colony formation in a concentration-dependent manner, reducing the number of colonies by $64.7 \% \pm 4.8 \%$ and $75.8 \% \pm 5.4 \%$ when applied in the form of Taxol and liposomes at $8 \mathrm{nM}$ PTX concentration, respectively. At a concentration of $20 \mathrm{nM}$, PTX almost completely prevented formation of colonies regardless of formulation, indicating that repeated liposome treatment was as effective as treatment with Taxol. Under the same conditions, PTX-free liposomal carriers did not affect the formation of colonies (data not shown). These results demonstrate that triglyceride-incorporated liposomes carrying PTX is equally potent as Taxol as an anticancer drug.

The in vivo anticancer efficacy of triglyceride-incorporated liposomal PTX was examined in SCC-7 tumor-bearing mice. As shown in Figure 7A, liposomal PTX reduced the tumor growth similar to Taxol. Probably PTX loading in triglyceride-incorporated liposomes circulated the bloodstream long without PTX leakage/precipitation, resulting in high accumulation of drug in the tumor via enhanced permeability and retention effect.

Body weight change was assessed as an indicator of safety including side effects and toxicities of treatment. Group of mice treated with Taxol underwent a temporary weight loss of $\sim 11 \%$ during treatment while no significant weight loss was observed by liposomal PTX treatment (Figure 7B). It indicates that the toxicity of Taxol, which may be associated with the use of Cremophor, could be overcome when PTX was loaded in triglyceride-incorporated liposomes. 
A

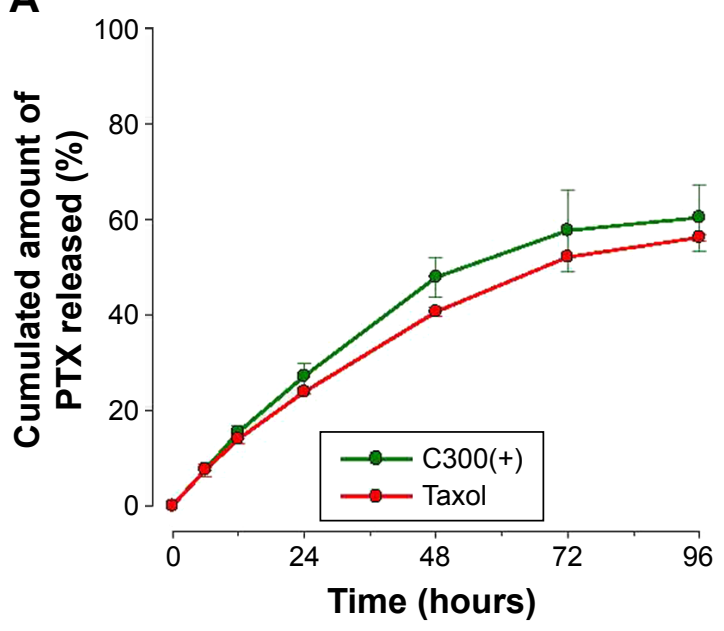

B

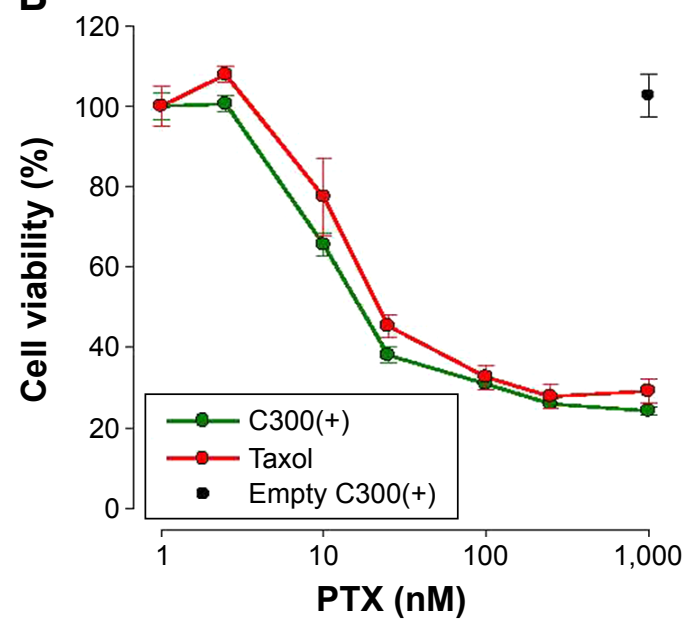

C
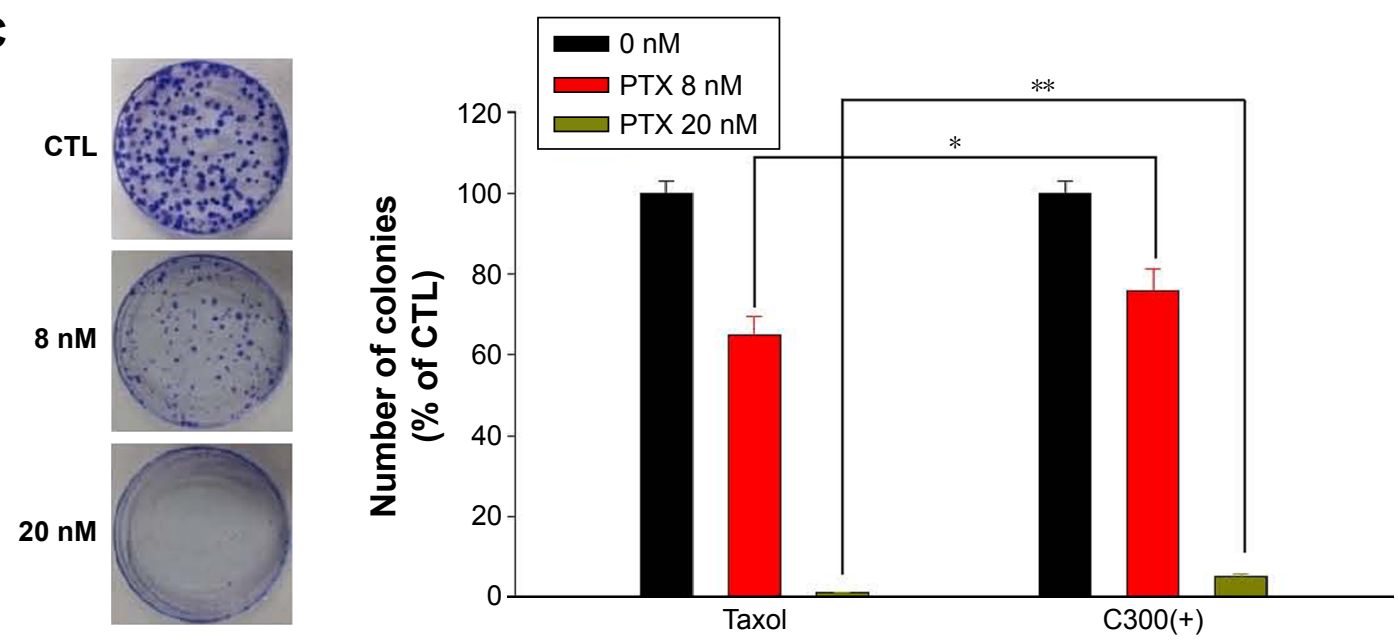

Figure 6 Release profile and in vitro anticancer efficacy of varying PTX formulations.

Notes: (A) Comparison of PTX release profile between liposomal formulation and Taxol ${ }^{\circledR}$. (B) Evaluation of anticancer efficacy of liposomal formulation in comparison with Taxol. Human $\mathrm{H} 460$ lung cancer cells treated with varying concentrations of PTX-loaded C300(+) liposomes or Taxol for 48 hours were subjected to MTT assay. (C) Comparison of antiproliferative effects assessed by colony formation assay. Mouse BI6-FI0 melanoma cells were treated for 8 days with different concentrations of PTX-loaded $\mathrm{C} 300(+)$ liposomes or Taxol. The number of colonies in the dish treated with saline was used as an index for a $100 \%$ (control) and this value was used to obtain the percentage colony numbers for other dishes. Left panel is the representative dishes following crystal violet staining of cell colonies treated with liposomal formulation $(n=4, * P<0.05, * * P<0.005$ compared to Taxol). PTX-loaded liposomes were prepared with a 7:1:0.4 of DMPC:CHOL:PE-PEG mixture with C300 ( $2 \mathrm{mg}$ per $42 \mu$ mole lipid).

Abbreviations: C300(-), Captex 300-free liposomes; C300(+), Captex 300-incorporated liposomes; CHOL, cholesterol; CTL, control; DMPC, I,2-dimyristoyl-snglycero-3-phosphocholine; empty C300(+), paclitaxel-free/Captex 300-incorporated liposomes; MTT, 3-(4,5-dimethylthiazol-2-yl)-2,5-diphenyltetrazolium bromide; PE-PEG, N-(Carbonyl-methoxypolyethyleneglycol 2000)-I, 2-distearoyl-sn-glycero-3-phosphoethanolamine; PTX, paclitaxel; SD, standard deviation.

Taxol is known to induce hemolysis after intravenous administration owing to the presence of Cremophor EL, ${ }^{41}$ a surfactant that can cause membrane damage. We assessed the hemolysis potential of the liposomal formulation in comparison with Taxol using RBCs. The percentage of hemolytic cells after 1-hour incubation with Taxol increased with increasing PTX concentration up to $350 \mathrm{nM}$ PTX, plateauing at a maximum of $69.7 \%$ (Figure $7 \mathrm{C}$ ). The concentration range of Taxol that induced hemolysis is consistent with that reported previously. ${ }^{42}$ Incubation with $350-1,050 \mathrm{nM}$ PTX as liposome-loaded formulations also induced hemolysis in a PTX concentration-dependent manner; however, the percentage of hemolytic cells induced by $350 \mathrm{nM}$ PTX in C300(+) liposomes was 5.3-fold lower than the same PTX concentration in a Taxol formulation. The greatest amount of hemolysis triggered by a liposome-loaded form, observed at 1,050 nM PTX, was still $<20 \%$, and was likely caused by PTX-RBC interactions ${ }^{43}$ Combined together, these data indicate that triglyceride-incorporated liposomes would cause less toxicity than Taxol after intravenous administration.

\section{Conclusion}

Earlier studies have highlighted the challenge of developing injectable PEGylated liposomes as a PTX carrier, a challenge 

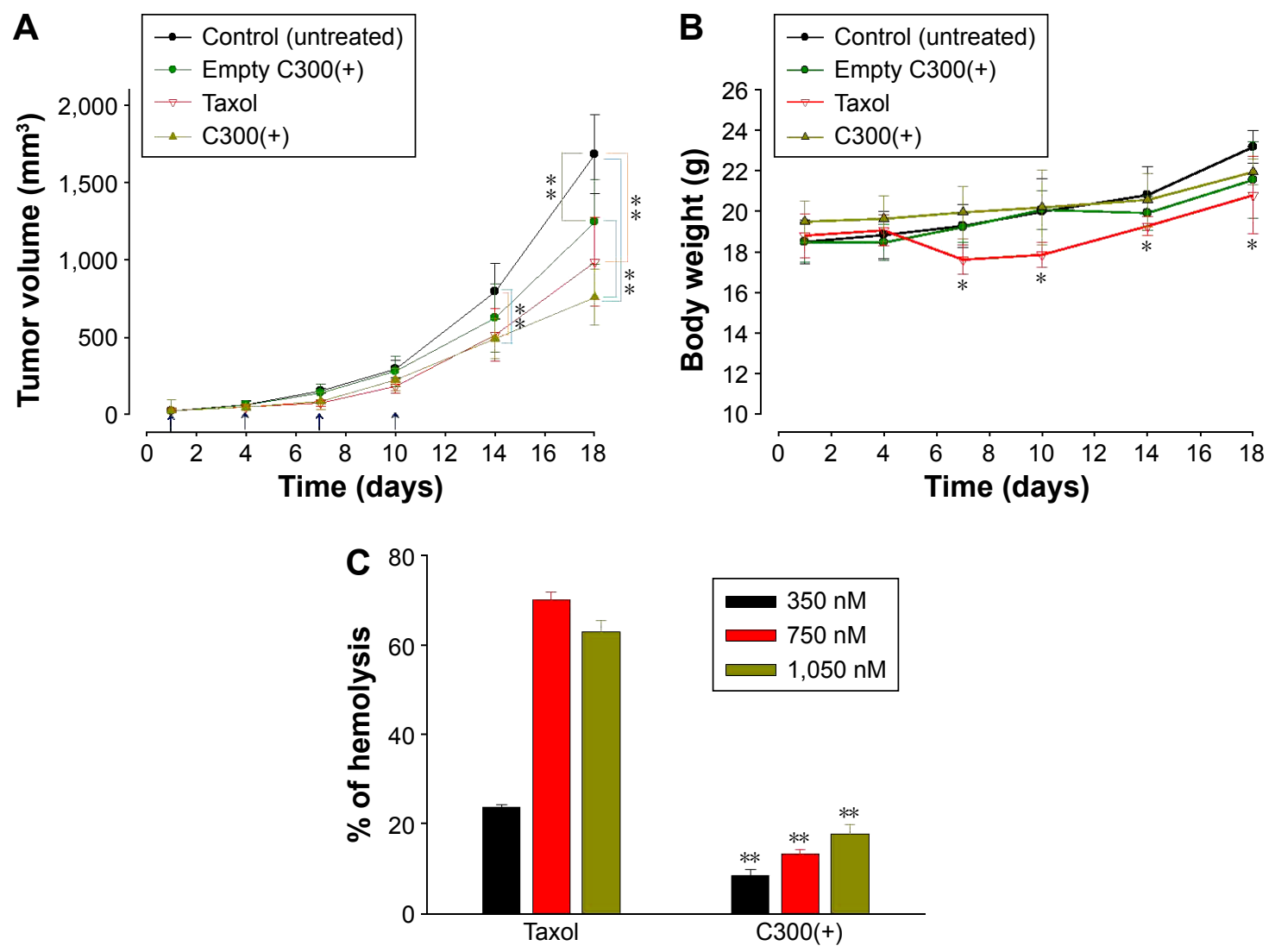

Figure 7 In vivo anticancer activity and hemolytic toxicity of PTX formulations.

Notes: (A) Anticancer activity of $\mathrm{C} 300(+)$ liposomes in comparison with Taxol. Tumor volume changes were determined after injection of formulations into the tail vein of each mouse ( $\mathrm{n}=6-7)$, $* * P<0.005$. The upward arrow represents administration time points. (B) Body weight changes of SCC7 tumor-bearing mice after injection. $* P<0.05$ compared to control. (C) Comparison of hemolytic toxicity of Taxol ${ }^{\circledR}$ and liposomal PTX formulation $(\mathrm{n}=3)$. $* * P<0.005$ compared to Taxol. PTX-loaded liposomes were prepared with a 7:1:0.4 of DMPC:CHOL:PE-PEG mixture with C300 (2 mg per $42 \mu$ mole lipid).

Abbreviations: C300(+), Captex 300-incorporated liposomes; CHOL, cholesterol; DMPC, I,2-dimyristoyl-sn-glycero-3-phosphocholine; PE-PEG, N-(Carbonylmethoxypolyethyleneglycol 2000)-I, 2-distearoyl-sn-glycero-3-phosphoethanolamine; PTX, paclitaxel; SD, standard deviation.

attributable to the limitations in liposomal stability caused by PTX loading. The data presented here demonstrate that triglyceride incorporation in PEGylated/saturated PC-based liposomes produced fine, homogeneous, and membrane filterable liposomes carrying PTX suitable for intravenous dosing. Triglyceride also functioned as a formulation stabilizer inhibiting the liposomal fusion and the PTX leakage during storage and thus triglyceride-incorporated liposomes could stably retain PTX up to 6-month storage. Further studies are warranted to reinforce the validity of triglyceride-incorporated liposomes as an injectable PTX dosage form for the treatment of cancer patients.

\section{Acknowledgments}

This work was supported by grants from the Basic Science Research Program through the National Research Foundation of Korea (NRF) funded by the Ministry of Education, Science and Technology (2012R1A1A2042768 and 2015R1A2A2A01005783). The negative staining TEM images were obtained at the Korea Basic Science Institute (KBSI).

\section{Disclosure}

The authors report no conflicts of interest in this work.

\section{References}

1. Goldspiel BR. Clinical overview of the taxanes. Pharmacotherapy. 1997;17(5 Pt 2):110s-125s.

2. Rowinsky EK, Eisenhauer EA, Chaudhry V, Arbuck SG, Donehower RC. Clinical toxicities encountered with paclitaxel (Taxol). Semin Oncol. 1993;20(4 Suppl 3):1-15.

3. Fjällskog ML, Frii L, Bergh J. Is Cremophor EL, solvent for paclitaxel, cytotoxic? Lancet. 1993;342(8875):873.

4. Dorr RT. Pharmacology and toxicology of Cremophor EL diluent. Ann Pharmacother. 1994;28(5 Suppl):S11-S14.

5. Yang T, Cui FD, Choi MK, et al. Liposome formulation of paclitaxel with enhanced solubility and stability. Drug Deliv. 2007;14(5): 301-308.

6. Stinchcombe TE. Nanoparticle albumin-bound paclitaxel: a novel Cremphor-EL-free formulation of paclitaxel. Nanomedicine (Lond). 2007;2(4):415-423.

7. Cline EN, Li MH, Choi SK, et al. Paclitaxel-conjugated PAMAM dendrimers adversely affect microtubule structure through two independent modes of action. Biomacromolecules. 2013;14(3): 654-664.

8. Danhier F, Lecouturier N, Vroman B, et al. Paclitaxel-loaded PEGylated PLGA-based nanoparticles: in vitro and in vivo evaluation. $J$ Control Release. 2009;133(1):11-17. 
9. Kim TY, Kim DW, Chung JY, et al. Phase I and pharmacokinetic study of Genexol-PM, a cremophor-free, polymeric micelle-formulated paclitaxel, in patients with advanced malignancies. Clin Cancer Res. 2004;10(11):3708-3716.

10. Green MR, Manikhas GM, Orlov S, et al. Abraxane, a novel Cremophor-free, albumin-bound particle form of paclitaxel for the treatment of advanced non-small-cell lung cancer. Ann Oncol. 2006;17(8): 1263-1268.

11. Yang T, Cui FD, Choi MK, et al. Enhanced solubility and stability of PEGylated liposomal paclitaxel: in vitro and in vivo evaluation. Int $J$ Pharm. 2007;338(1-2):317-326.

12. Sen K, Mandal M. Second generation liposomal cancer therapeutics: transition from laboratory to clinic. Int J Pharm. 2013;448(1):28-43.

13. Zhang JA, Anyarambhatla G, Ma L, et al. Development and characterization of a novel Cremophor EL free liposome-based paclitaxel (LEPETU) formulation. Eur J Pharm Biopharm. 2005;59(1):177-187.

14. Sharma A, Straubinger RM. Novel taxol formulations: preparation and characterization of taxol-containing liposomes. Pharm Res. 1994; 11(6):889-896.

15. Kan P, Tsao CW, Wang AJ, Su WC, Liang HF. A liposomal formulation able to incorporate a high content of paclitaxel and exert promising anticancer effect. J Drug Deliv. 2011;2011:629234.

16. Kannan V, Balabathula P, Divi MK, Thoma LA, Wood GC. Optimization of drug loading to improve physical stability of paclitaxel-loaded long-circulating liposomes. J Liposome Res. 2015;25(4):308-315.

17. Koudelka S, Turanek-Knotigova P, Masek J, et al. Liposomes with high encapsulation capacity for paclitaxel: Preparation, characterisation and in vivo anticancer effect. J Pharm Sci. 2010;99(5):2309-2319.

18. Crosasso P, Ceruti M, Brusa P, Arpicco S, Dosio F, Cattel L. Preparation, characterization and properties of sterically stabilized paclitaxelcontaining liposomes. J Control Release. 2000;63(1-2):19-30.

19. Immordino ML, Brusa P, Arpicco S, Stella B, Dosio F, Cattel L. Preparation, characterization, cytotoxicity and pharmacokinetics of liposomes containing docetaxel. J Control Release. 2003;91(3):417-429.

20. Ye L, He J, Hu Z, et al. Antitumor effect and toxicity of Lipusu in rat ovarian cancer xenografts. Food Chem Toxicol. 2013;52:200-206.

21. Slingerland M, Guchelaar HJ, Rosing H, et al. Bioequivalence of liposome-entrapped paclitaxel easy-to-use (LEP-ETU) formulation and paclitaxel in polyethoxylated castor oil: a randomized, twoperiod crossover study in patients with advanced cancer. Clin Ther. 2013;35(12):1946-1954.

22. Fasol U, Frost A, Buchert M, et al. Vascular and pharmacokinetic effects of EndoTAG-1 in patients with advanced cancer and liver metastasis. Ann Oncol. 2012;23(4):1030-1036.

23. Mattjus P, Slotte JP. Does cholesterol discriminate between sphingomyelin and phosphatidylcholine in mixed monolayers containing both phospholipids? Chem Phys Lipids. 1996;81(1):69-80.

24. Payton NM, Wempe MF, Betker JL, Randolph TW, Anchordoquy TJ. Lyophilization of a triply unsaturated phospholipid: effects of trace metal contaminants. Eur J Pharm Biopharm. 2013;85(2):306-313.

25. Lehtonen JY, Kinnunen PK. Poly(ethylene glycol)-induced and temperature-dependent phase separation in fluid binary phospholipid membranes. Biophys J. 1995;68(2):525-535.

26. Hong RL, Huang CJ, Tseng YL, et al. Direct comparison of liposomal doxorubicin with or without polyethylene glycol coating in C-26 tumorbearing mice: is surface coating with polyethylene glycol beneficial? Clin Cancer Res. 1999;5(11):3645-3652.

International Journal of Nanomedicine

\section{Publish your work in this journal}

The International Journal of Nanomedicine is an international, peerreviewed journal focusing on the application of nanotechnology in diagnostics, therapeutics, and drug delivery systems throughout the biomedical field. This journal is indexed on PubMed Central, MedLine, CAS, SciSearch $®$, Current Contents $\AA /$ Clinical Medicine,
27. Hong SS, Kim SH, Lim SJ. Effects of triglycerides on the hydrophobic drug loading capacity of saturated phosphatidylcholine-based liposomes. Int J Pharm. 2015;483(1-2):142-150.

28. Hong S-S, Lim S-J. Laboratory scale production of injectable liposomes by using cell disruptor to avoid the probe sonication process. J Pharm Investig. 2015;45(1):73-78.

29. Kang DI, Lee S, Lee JT, et al. Preparation and in vitro evaluation of anti-VCAM-1-Fab'-conjugated liposomes for the targeted delivery of the poorly water-soluble drug celecoxib. J Microencapsul. 2011; 28(3):220-227.

30. Lee EH, Hong SS, Kim SH, Lee MK, Lim JS, Lim SJ. Computed tomography-guided screening of surfactant effect on blood circulation time of emulsions: application to the design of an emulsion formulation for paclitaxel. Pharm Res. 2014;31(8):2022-2034.

31. Kang SN, Hong SS, Lee MK, Lim SJ. Dual function of tributyrin emulsion: solubilization and enhancement of anticancer effect of celecoxib. Int J Pharm. 2012;428(1-2):76-81.

32. Kim S, Kim JY, Huh KM, Acharya G, Park K. Hydrotropic polymer micelles containing acrylic acid moieties for oral delivery of paclitaxel. J Control Release. 2008;132(3):222-229.

33. Lim SJ, Lee MK, Kim CK. Altered chemical and biological activities of all-trans retinoic acid incorporated in solid lipid nanoparticle powders. J Control Release. 2004;100(1):53-61.

34. Liang H, Yang Q, Deng L, Lu J, Chen J. Phospholipid-Tween 80 mixed micelles as an intravenous delivery carrier for paclitaxel. Drug Dev Ind Pharm. 2011;37(5):597-605.

35. Romberg B, Oussoren C, Snel CJ, Hennink WE, Storm G. Effect of liposome characteristics and dose on the pharmacokinetics of liposomes coated with poly(amino acid)s. Pharm Res. 2007;24(12):2394-2401.

36. Hinrichs WL, Mancenido FA, Sanders NN, et al. The choice of a suitable oligosaccharide to prevent aggregation of PEGylated nanoparticles during freeze thawing and freeze drying. Int J Pharm. 2006;311(1-2): 237-244.

37. Campbell RB, Balasubramanian SV, Straubinger RM. Influence of cationic lipids on the stability and membrane properties of paclitaxelcontaining liposomes. J Pharm Sci. 2001;90(8):1091-1105.

38. Muller RH, Heinemann S. Fat emulsions for parenteral nutrition. I: evaluation of microscopic and laser light scattering methods for the determination of the physical stability. Clin Nutr. 1992;11(4):223-236.

39. Kannan V, Balabathula P, Thoma LA, Wood GC. Effect of sucrose as a lyoprotectant on the integrity of paclitaxel-loaded liposomes during lyophilization. J Liposome Res. 2015;25(4):270-278.

40. Lu J, Chuan X, Zhang H, et al. Free paclitaxel loaded PEGylated-paclitaxel nanoparticles: preparation and comparison with other paclitaxel systems in vitro and in vivo. Int J Pharm. 2014;471(1-2):525-535.

41. Liu F, Park JY, Zhang Y, et al. Targeted cancer therapy with novel high drug-loading nanocrystals. J Pharm Sci. 2010;99(8):3542-3551.

42. Tang B, Fang G, Gao Y, et al. Liprosomes loading paclitaxel for braintargeting delivery by intravenous administration: in vitro characterization and in vivo evaluation. Int J Pharm. 2014;475(1-2):416-427.

43. Harisa GI. Naringin mitigates erythrocytes aging induced by paclitaxel: an in vitro study. J Biochem Mol Toxicol. 2014;28(3):129-136. 